\title{
Áreas organizativas específicas y circuitos preferentes para patologías prevalentes en urgencias
}

\section{Specific organisational areas and preferential circuits for prevalent pathologies in emergency care}

\author{
M. Sánchez ${ }^{1}$, I. Santiago ${ }^{2}$
}

\section{RESUMEN}

El incremento de la demanda y las habituales situaciones de colapso de los Servicios de Urgencias Hospitalarios (SUH) obliga a continuos cambios organizativos en busca de un óptimo funcionamiento y una asistencia de mayor calidad.

Los principales cambios se han basado en la implantación de diferentes sistemas de triaje, a fin de estructurar la atención en función de la urgencia, y el cambio adaptativo en la asistencia médica mediante diferentes fórmulas, como la derivación sin visita, las áreas de visita rápida (fast-track areas) y los circuitos asistenciales específicos, como las unidades de dolor torácico, de código ictus o de código sepsis, entre las más extendidas en los SUH.

Por otro lado, los SUH se han visto obligados a buscar y utilizar métodos que favorezcan el flujo de salida de los pacientes desde los propios servicios, la mayor parte de las veces entorpecida por la mala gestión de camas hospitalarias por parte de las administraciones sanitarias. Para ello se utilizan diferentes alternativas a la hospitalización convencional como son: unidades de observación, unidades de estancia corta, hospitales de día y hospitalización domiciliaria.

Palabras clave. Áreas específicas. Circuitos asistenciales. Alternativas a la hospitalización convencional.

\begin{abstract}
The increase in demand and the normal situations of overload of the Hospital Emergency Department (HED) make continuous organisational changes compulsory in the search for optimum working and greater quality of care.

The main changes have been based on the implementation of different triage systems, with the aim of structuring care according to urgency, and adaptive change in the medical service through different formulae, such as referrals without consultation, fast-track areas and specific care circuits, such as units for thoracic pain, stoke code or sepsis code, amongst the most widespread in HED.

On the other hand, HEDs have been forced to seek and use methods that favour the discharge of patients, often hindered by poor management of hospital beds by the health administrations. Hence, different alternatives to hospitalisation are suggested, such as: observation units, short stay units, day hospitals and hospitalisation at home.
\end{abstract}

Key words. Specific areas. Care circuits. Alternatives to conventional hospitalisation.

\section{Secció d’Urgències Medicina. Àrea} d’Urgències. Hospital Clínic. Barcelona.

2. Servicio de Análisis y Control Asistencial. Servicio Navarro de Salud-Osasunbidea. Pamplona.

\author{
Correspondencia \\ Dr. Miquel Sánchez \\ Secció d`Urgències Medicina. Àrea d`Urgències \\ Hospital Clínic \\ Villarroel, 170 \\ 08036 Barcelona \\ E-mail: msanchez@clinic.ub.es
}




\section{INTRODUCCIÓN}

Los servicios de urgencias hospitalarios (SUH) constituyen el vértice de la pirámide del sistema de atención médica urgente. La frecuentación en los SUH se ha incrementado en los últimos años ${ }^{1}$ presentando, incluso, patrones diferentes según el origen de los pacientes ${ }^{2}$. En España, en el año 2004, se atendieron en total 23.654.303 visitas urgentes en los hospitales, con un incremento total del $45,5 \%$ respecto a las que se atendían 10 años antes ${ }^{3}$. Este incremento, en la mayoría de ocasiones, no se ha acompañado de un aumento paralelo ni en estructura ni en recursos humanos de los $\mathrm{SUH}^{4}$. Como consecuencia, con excesiva frecuencia se acumulan esperas superiores a las que serían deseables con el consiguiente deterioro en la calidad ${ }^{5}$ y efectividad $^{6}$. A estos períodos en los que el SUH se encuentra desbordado se les denomina comúnmente saturación o colapso y, con frecuencia, alimentan ecos mediáticos negativos hacia los mismos ${ }^{7}$.

Los SUH han reaccionado clásicamente a esta situación con discursos que ponían énfasis en la gran cantidad de visitas inadecuadas que debían afrontar por motivos diversos, incluso, por déficit de otros niveles asistenciales del sistema sanitario público español ${ }^{8}$. Ello, unido al hecho incuestionable que la parte proporcionalmente más importante del problema radica no en determinantes externos (fundamentalmente la demanda de asistencia por parte de los usuarios) sino en determinantes internos (fundamentalmente la permanencia de los pacientes ingresados en urgencias ${ }^{9}$ ), conduce a una actitud más proactiva en los SUH, que han buscado soluciones desde dentro. Se han producido esfuerzos imaginativos individuales que han concluido en cambios discrecionales como respuesta a requerimientos locales de un determinado SUH. El buen resultado obtenido por algunos de ellos tendría que haber favorecido su asunción por parte de la administración y la explícita implantación en ambientes y entornos similares a aquellos en los que se ha demostrado beneficio. El objetivo de la presente revisión es la presentación y des- cripción de algunas de las soluciones más representativas que, desde los $\mathrm{SUH}$, se han impulsado (cuando no liderado) durante los últimos años para sobrevivir a las situaciones de colapso.

\section{CAMBIOS ORGANIZATIVOS DENTRO DEL PROPIO SERVICIO}

Todas aquellas medidas o procesos diseñados para hacer el SUH más eficiente en tiempo y/o en recursos, se encuadran dentro de este epígrafe y se conocen como cambios organizativos del propio SUH.

Entre ellas, cabe destacar: el triaje estructurado, el triaje avanzado, el triaje multidisciplinar, la derivación sin visita, las áreas de visita rápida o fast-track y los circuitos asistenciales específicos, ya sea funcionales o estructurales.

\section{TRIAJE ESTRUCTURADO}

El triaje es un proceso de valoración clínica preliminar que ordena a los pacientes antes de la valoración diagnóstica y terapéutica, de forma que los pacientes más graves son tratados los primeros. Este proceso de priorización, cuando se lleva a cabo a partir de escalas válidas, útiles, relevantes y reproducibles se denomina triaje estructurado. En estos momentos existen 5 sistemas de triaje estructurado: el ATS (Australasian Triage Scale), el CTAS (Canadian Triage and Acuity Scale), el MTS (Manchester Triage Scale), el ESI (Emergency Severity Index) y el MAT (Model Andorrà de Triatge $)^{10}$. Este último ha sido adoptado por la Sociedad Española de Medicina de Urgencias y Emergencias (SEMES) como el estándar de triaje estructurado español con la denominación de Sistema Español de Triaje (SET). Los resultados obtenidos tras la aplicación de alguno de los triajes anteriores sobre el SUH son: la identificación rápida y eficiente de los pacientes que presentan una enfermedad que pone en peligro su vida con el objetivo de priorizar su asistencia; determinar el área de tratamiento más adecuada para los pacientes, ayudando a la descongestión de las mis- 
mas; gestionar debidamente las esperas, revalorando e informando a familiares y pacientes de una forma precisa; proporcionar información que permita conocer y comparar casuística de los $\mathrm{SUH}$, con la finalidad de optimizar recursos y mejorar la eficiencia ${ }^{10}$.

\section{TRIAJE AVANZADO}

Esta modalidad contempla, en un triaje estructurado, la posibilidad, de acuerdo a la priorización asignada y a protocolos específicos especialmente elaborados para enfermería, de realizar, antes de la visita convencional, determinadas pruebas complementarias, básicamente analíticas y radiología simple o, incluso, acciones terapéuticas como canalizar vías periféricas, administrar analgesia, inmovilizar con yeso... Bajo estas premisas es fácil suponer que determinados pacientes de baja gravedad se visitan con mayor rapidez ${ }^{11}$.

\section{TRIAJE MULTIDISCIPLINAR}

Representa un escalón más respecto al anterior. Se entiende como triaje multidisciplinar aquel que incluye, entre el personal sanitario que lo realiza, un equipo en el que, como mínimo, participa un médico y una enfermera $^{12}$. En algunas ocasiones, y para acelerar registros administrativos y trámites de llegada a un SUH, se incluye personal administrativo. También, para facilitar el desplazamiento de pacientes, puede sumarse un auxiliar sanitario. Aparte de la valoración inicial del paciente que, en este caso, la realiza el médico, la idea del triaje multidisciplinar consiste en la realización de pruebas diagnósticas básicas (hemograma, bioquímica y radiología simple) antes de que el paciente entre en el box de visita. El médico, una vez recibe las pruebas, puede optar por dar de alta al paciente, si así procede, o visitarle de la forma convencional pero con la ventaja de disponer ya de las exploraciones complementarias. Lógicamente, aunque se invierte lo que podríamos denominar la visita "académica" del paciente, los tiempos asistenciales se reducen considerablemente y las esperas también disminuyen. Esta experiencia, probada a tiempo parcial (diariamente de 10 a 20 horas) en el Inova Fairfax Hospital en Washington DC (USA) permitió reducir el tiempo medio de estancia global en urgencias en casi 4 horas, a la vez que aumentó la calidad percibida de los pacientes y su predisposición a volver a dicho SUH en casi un $100 \%$. En la actualidad esta modalidad se lleva a cabo en algunos hospitales de Melburne (Australia) ${ }^{13}$.

\section{DERIVACIÓN SIN VISITA}

Partiendo de la hipótesis, tan empleada por otro lado, de que un gran número de visitas que acuden a los SUH son inadecuadas, no resulta descabellado pensar que, desde el mismo $\mathrm{SUH}$, podrían redireccionarse hacia el nivel asistencial más pertinente. Los resultados tendrían que ser evidentes en la descongestión del servicio. La primera parte de la hipótesis se ha confirmado en un reciente trabajo donde se demuestra que alrededor de un $15 \%$ de pacientes que acuden a los SUH por patología médica pueden ser derivados sin visita de forma efectiva a otros niveles de la red asistencial donde se atenderá su consulta. El riesgo de esta política cabe considerarlo muy bajo puesto que menos de un $2 \%$ de estos pacientes retornarán al SUH y menos del $0,2 \%$ serán ingresados. Aunque se detecta cierto escepticismo por parte del usuario en el momento de proponerse la derivación, su percepción final respecto al trato médico, al grado de resolución del problema y la satisfacción global es buena $^{14}$. Desgraciadamente los autores no valoraron el impacto que esta medida tenía sobre los parámetros de efectividad y calidad del SUH, en definitiva, si la medida era útil para aliviar la presión asistencial de los mismos.

\section{ÁREAS DE VISITA RÁPIDA}

La creación de áreas de visita rápida o fast-track areas es una manera de incrementar el flujo de pacientes que un determina- 
do SUH puede visitar simultáneamente ${ }^{15}$. Se trata de espacios físicos dentro del propio servicio donde se atienden a los pacientes de menor gravedad, aquellos que, en general, pueden ser dados de alta rápidamente con un mínimo, si no ninguna, exploración complementaria. Normalmente, en los países anglosajones, personal no médico especialmente cualificado es responsable del área y, por lo tanto, de resolver este tipo de pacientes. En el argot anglosajón se les conoce como nurse practitioner o physician asistant y, en nuestro entorno, sería el equivalente a enfermeras con probada experiencia en urgencias que realizarían un aprendizaje especial y específico de 2 años enfocado a explorar, diagnosticar y tratar a estos pacientes. Se ha demostrado que estas áreas con este tipo específico de personal sanitario son capaces de disminuir el tiempo de espera, el tiempo total de estancia en urgencias y el porcentaje de pacientes que se marchan del SUH antes de ser visitados, sin detrimento en la calidad técnica dispensada ${ }^{15}$.

\section{CIRCUITOS ASISTENCIALES ESPECÍFICOS}

\section{Unidades de dolor torácico}

En la actualidad, la probabilidad de que cualquier paciente que acude a un SUH tenga que esperar para ser visitado es alta. Los pacientes con dolor torácico, que pueden suponer hasta el $5 \%$ de las visitas $^{16}$, se consideran en el grupo de atención que requiere atención inmediata, ya que la identificación precoz de un síndrome coronario agudo (SCA) y el inicio del tratamiento de reperfusión, cuando está indicado, a la mayor brevedad tiene importantes implicaciones pronósticas ${ }^{17}$. Es por ello que las unidades de dolor torácico (UDT) han proliferado rápidamente en Estados Unidos. Conceptualmente, éstas pueden ser funcionales o estructurales. De hecho, la implantación y cumplimiento de un protocolo específico que sirva para identificar con prontitud los pacientes con un SCA y descartarlo eficientemente en aquellos que no lo padecen, de forma que se reduzcan los ingresos inadecuados o las altas erróneas, es una UDT funcional. Aunque las UDT estructurales no han gozado de una aceptación generalizada en nuestro entorno, constituyen una manera eficiente de afrontar la atención de los pacientes con dolor torácico de una forma equitativa ${ }^{18} \mathrm{y}$ consiguen una clara mejoría en los tiempos asistenciales de estos pacientes ${ }^{16}$.

\section{Código ictus}

Con el desarrollo de tratamientos específicos fibrinolíticos y endovasculares en el ictus ligados al tiempo de evolución del fenómeno isquémico agudo, en determinados hospitales se han desarrollado estrategias, la mayoría funcionales, de circuitos asistenciales propios para la atención a esta patología en el tiempo preciso. En la actualidad, ya sea por su juventud o por imposibilidades ligadas a la ineficiencia de estos circuitos o por la elevada exigencia de estos protocolos, el resultado de las mismas es pobre, puesto que no se consigue incrementar de forma significativa el porcentaje de pacientes sometidos a estos tratamientos ${ }^{19}$.

\section{Código sepsis}

Recientemente se ha asistido a la concienciación de la conveniencia de instaurar un tratamiento precoz y agresivo en los pacientes con sepsis, lo que se ha denominado "código sepsis». Este nuevo circuito funcional persigue homogeneizar e instaurar de forma precoz un tratamiento antibiótico y hemodinámica adecuado. La implantación del mismo ha reflejado resultados destacados que deberían confirmarse en el futuro ${ }^{20}$.

\section{ALTERNATIVAS A LA HOSPITALIZACIÓN CONVENCIONAL}

La fuerte presión de urgencias que viven los hospitales cuando el SUH está saturado de pacientes ingresados, condiciona una disminución en la oferta de camas hospitalarias para la realización de ingresos, 
básicamente quirúrgicos, programados. Este hecho ha llevado, en algunos centros, a un rediseño de los recursos de los SUH que, necesariamente, han reordenado y mejorado su trabajo clínico adaptándolo a las fluctuaciones horarias, diarias e, incluso, estacionales, de la demanda. Nacen, así, las alternativas a la hospitalización convencional para diagnosticar y tratar los pacientes que acuden a un SUH de forma más eficiente, evitando el ingreso hospitalario convencionalmente entendido. Se consideran alternativas a la hospitalización convencional a las unidades de observación (UO), las unidades de estancia corta (UEC), los hospitales de día (HD) y la hospitalización a domicilio (HAD).

\section{Unidades de observación}

La necesidad de una UO ligada a un SUH aparece hace más de 40 años. En 1989, la British Association of Accident and Emergency Medicine (BAEM) recomendó su extensión a todo SUH, aconsejando la instauración de una cama de observación por cada 5.000 urgencias anuales atendidas. Desde entonces y hasta el momento actual, sin embargo, la terminología en torno al concepto de UO es confusa. En efecto, en la literatura, términos como salas de observación, salas de admisión de urgencias, salas de tratamiento e, incluso, UEC, se han utilizado de forma indistinta. Es por ello que en nuestro medio, a fin de delimitar conceptos, se considere una UO al recurso que se especifica en el «Manual de Estándares de Acreditación de los Servicios de Urgencias Hospitalarios" y que se concreta en: espacio asistencial con una capacidad mínima para atender el $10 \%$ de la demanda urgente diaria, con unos protocolos de ingreso, derivación y alta claramente escritos y con una estancia máxima de 24 horas $^{21}$. Entre las ventajas de estas áreas se encuentra una reducción significativa del tiempo global de estancia, un mayor grado de satisfacción en los pacientes que la utilizan, una disminución de la carga de trabajo total del SUH, y un mecanismo de control para evitar altas inadecuadas. Entre las desventajas, la más perjudicial y la que condiciona su funcionamiento en el futuro, radica en la aceptación de ingresos hospitalarios con imposibilidad de drenaje que acaba colapsando el recurso. En nuestro entorno, estudios de parecidas características sobre la rentabilidad de las unidades de observación ${ }^{22}$, han conducido a conclusiones superponibles, sobre todo cuando se ha analizado para patologías concretas como la pielonefritis aguda ${ }^{23} \mathrm{o}$ el flutter auricular ${ }^{24}$.

\section{Unidades de estancia corta}

Conceptualmente son áreas de hospitalización dependientes de los SUH, con una cantidad variable de camas, en las que ingresan determinados pacientes con determinadas patologías bajo protocolos estrictos de diagnóstico y tratamiento y con una estancia total que no debe superar, por lo general, los 2-3 días. Un aspecto importante a tener en cuenta es que, aunque de nombre parecido, si no idéntico, a otras áreas que nacen dependientes de algún servicio hospitalario, presentan una característica diferencial propia: el personal sanitario está adscrito al SUH y, por lo tanto, están operativas las 24 horas del día, a diferencia de otras UEC hospitalarias que funcionan en horario hospitalario estándar. Esto permite una mayor rotación enfermo/ cama y, por lo tanto, un uso más eficiente del recurso ${ }^{25}$. Además se ha demostrado que este aspecto positivo en su gestión se obtiene a expensas de una seguridad y satisfacción para el paciente que, cuando menos, es superponible a la hospitalización convencional $^{26,27}$.

\section{Hospitales de día}

Estas áreas concretas se crearon a finales del siglo pasado en nuestro país adscritas a un servicio hospitalario concreto para facilitar las visitas y seguimiento de los pacientes de una forma más compleja a la que se venía haciendo hasta la fecha en las clásicas consultas externas. Así, pronto proliferaron los hospitales de día que visitaban pacientes con sida que acudían a rea- 
lizar complejos tratamientos profilácticos de determinadas infecciones oportunistas, aquéllos que trataban a pacientes oncológicos o hematológicos que precisaban de tratamientos quimioterápicos o soporte transfusional frecuente $o$, más recientemente, los que seguían pacientes diabéticos con complicaciones menores tratables bajo este nuevo régimen. Su utilidad y sentido en los SUH está por dilucidar ya que, aunque algunos hospitales australianos disponen de ellos ${ }^{13}$, no han publicado su experiencia.

\section{Hospitalización a domicilio}

La hospitalización a domicilio se define como una alternativa asistencial capaz de realizar en el domicilio procedimientos diagnósticos, terapéuticos y cuidados similares a los dispensados en los hospitales. La actividad se lleva a cabo por profesionales especializados, durante un período de tiempo limitado, a pacientes que de otra manera habrían precisado atención en un hospital de agudos en cualquiera de sus áreas. Éste es uno de sus aspectos diferenciales respecto a la asistencia domiciliaria basada en la atención primaria, orientada hacia los cuidados a largo plazo, las medidas preventivas y la educación para la salud.

La estancia media de la HAD debe ser similar a la de un servicio de un hospital de agudos. En su cartera de servicios se incluye la posibilidad de trasladar, de forma temprana a sus hogares, a pacientes agudos, crónicos reagudizados, posquirúrgicos, traumatológicos o a enfermos en situación terminal con descompensación de síntomas.

Tradicionalmente estas unidades no han dependido de los SUH, pero la situación actual ha obligado a la asunción de nuevas funciones que garanticen cierta descompresión de los mismos. Al igual que en las UEC gestionadas directamente desde los SUH, se ha podido identificar ciertos beneficios ausentes en la HAD de visión más clásica, a la vez de una gestión más eficiente del recurso ${ }^{28}$.

\section{CONSIDERACIONES FINALES}

En la presente revisión se ha efectuado una descripción de la mayoría de los esfuerzos adaptativos y de supervivencia que los SUH han "inventado" o "improvisado" para poder seguir llevando a término su misión, que no es otra que la de atender a los pacientes que demandan asistencia urgente. Por ello, un análisis frío de esta situación lleva a darnos cuenta de que gran parte de lo hasta aquí expuesto representa una huída hacia delante que poco o nada tiene que ver con esta la misión. En efecto, la ineficiencia hospitalaria que, en porcentaje, es la causa más importante del estado que en la actualidad viven los SUH en España, sigue campando a sus anchas. Así, como las altas no se ofrecen ni en cantidad ni en adecuación horaria de forma sincrónica a las necesidades reales de los SUH, éstos acaban por asumir funciones que van más allá de su auténtica misión. Se intenta corregir las ineficiencias solicitando camas de hospitalización (UEC), ingresando y controlando pacientes en domicilio (HAD) y, aún alguno, aventurándose con hospitales de día. Cada cual tiene su misión y su responsabilidad y, en este juego, la del hospital está perfectamente identificada. Por ello, algunos han intentado adaptarse creando salas de preingreso que vienen a paliar, temporalmente, la asincronía de las altas hospitalarias con las necesidades reales de urgencias. Los más osados, además, han incorporado salas de alta, a donde son dirigidos los pacientes que serán dados de alta desde las salas del hospital, en espera del informe, de la familia o de cualquier otro trámite que dificulte la ocupación temprana de esa cama por un ingreso de urgencias.

La actividad de urgencias es programable ${ }^{29} \mathrm{y}$, cuando se dispone de camas de hospitalización suficientes, no se deteriora hasta el punto de comprometer la calidad asistencial ${ }^{30}$. Por ello, resulta sorprendente que desde la administración, últimos depositarios y responsables de unos recursos finitos que no deben malgastar, no se apueste de forma valiente por las iniciativas que han probado su efi- 
ciencia, implantándolas y generalizándolas al resto de $\mathrm{SUH}$, a la vez que lideran y promueven un cambio claro en la gestión de las camas hospitalarias más acorde con la medicina del siglo XXI.

\section{BIBLIOGRAFÍA}

1. Zaragoza M, Calvo C, SaAd T, Morán FJ, San José S, HERnÁNDEZ P. Evolución de la frecuentación en un servicio de urgencias hospitalario. Emergencias 2009; 21: 339-345.

2. Font I, Izquierdo A, Puiguriguer J. Análisis de las consultas a un servicio de urgencias hospitalario según el origen de los pacientes. Emergencias 2009; 21: 262-268.

3. http://www.msc.es/gabinetePrensa/notaPrensa/desarrolloNotaPrensa.jsp?id=760 (accedido el 14/07/2007).

4. Moreno E. ¿Y si adaptáramos los servicios hospitalarios de urgencias a la demanda social y no a las necesidades de salud? Emergencias 2008; 20: 276-284.

5. Miró O, Sánchez M, Coll-Vinent B, Millá J. Indicadores de calidad en urgencias: comportamiento en relación con la presión asistencial. Med Clin (Barc) 2001; 116: 92-97.

6. Sánchez M, Smally AJ. Comportamiento de un servicio de urgencias según el día de la semana y el número de visitas. Emergencias 2007; 19: 319-322.

7. Requena J. Repercusión mediática de los servicios de urgencias. Emergencias 2008; 20: 187-190.

8. SÁNCHEz M. ¿Urgencias inadecuadas o recursos insuficientes? Med Clin (Barc) 2004; 123 : 619-620.

9. Sánchez M, Miró O, Coll-Vinent B, Bragulat E, Espinosa G, Gómez-Angelats E et al. Saturación del servicio de urgencias: factores asociados y cuantificación. Med Clin (Barc) 2003; 121: 167-172.

10. Gómez JimÉnEz J. Urgencia, gravedad y complejidad: un constructo teórico de la urgencia basado en el triaje estructurado. Emergencias 2006; 18: 156-164.

11. Lee K, Wong T, Chan R, Lau CC, Fu YK, Fung KH. Accuracy and efficiency of $\mathrm{X}$-ray requests initiated by triage nurses in an accident and emergency department. Accid Emerg Nurs 1996; 4: 179-181.

12. Martín-SÁnchez FJ, González-Del Castillo J, ZAmorano J, Candel FJ, González-Armengol JJ, VILLARRoEl $\mathrm{P}$ et al. El facultativo, un elemento necesario en el triaje de un servicio de urgencias en un hospital terciario. Emergencias 2008; 20: 41-47.

13. McD Taylor D, Bennett DM, Cameron PA. A paradigm shift in the nature of care provision in emergency departments. Emerg Med J 2004; 21: 681-684.

14. Miró O, Salgado E, Tomás S, Espinosa G, Estrada C, MARTi C et al. Derivación sin vista desde los servicios de urgencias hospitalarios: cuantificación, riesgos y grado de satisfacción. Med Clin (Barc) 2006; 126: 88-93.

15. Sánchez M, Smally AJ, Grant RJ, Jacobs LM. Effects of a fast-track area on emergency department performance. J Emerg Med 2006; 31: 117-120.

16. Bragulat E, López B, Miró O, Coll-Vinent B, JiméNEz S, APARICıO MJ et al. Análisis de la actividad de una unidad estructural de dolor torácico en un servicio de urgencias hospitalario. Rev Esp Cardiol 2007; 60: 276-284.

17. Bayón J, Alegría E, Bosch X, Cabadés A, Iglesias I, JiMÉNEZ NÁCHER JJ et al. Chest pain units. Organization and protocol for the diagnosis of acute coronary syndromes. Rev Esp Cardiol 2002; 55: 143-154.

18. Riesgo A, Bragulat E, López-Barbeito B, Sánchez M, Miró O. Aproximación diagnóstica al dolor torácico en urgencias: ¿existen diferencias entre mujeres y hombres? Emergencias 2008; 20: 399-404.

19. Gómez-Angelats E, Bragulat E, Obach V, GómezChoco M, SÁnchez M, Miró O. Resultados alcanzados con la puesta en marcha del circuito "código ictus" en un gran hospital: papel de urgencias y análisis de la curva de aprendizaje. Emergencias 2009; 21: 105-113.

20. Aguirre A, Echarte JL, Minguez S, Supervía A, Skaf E, Campodarve I. Implementación de un "código sepsis grave" en un servicio de urgencias. Emergencias 2009; 21: 255-261.

21. Sociedad Española de Medicina de Urgencias y Emergencias. Estándares de acreditación para servicios de urgencias hospitalarios. $1^{\mathrm{a}}$ ed. Madrid: Edicomplet, 2004.

22. Estella A, Pérez-Bello L, Sánchez JI, Toledo MD, Del Águila D. Actividad asistencial en la unidad de observación de un hospital de segundo nivel. Emergencias 2009; 21: 95-98.

23. Lluís M, Miró O, Perea M, Pedrol E, Mijana M, RODELLAR T et al. Evolución de las pacientes con pielonefritis aguda no complicada tras su atención inicial y alta directa desde un servicio de urgencias hospitalario. Emergencias 2009; 21: 325-332. 
24. Aldea-Molina E, Gómez J, Royo R, Rodrigo G, RIVAS M, LLERA R. Sala de observación de un servicio de urgencias: un lugar adecuado para el manejo del flutter auricular. Emergencias 2008; 20: 101-107.

25. Juan A, Salazar A, Álvarez A, Pérez JR, García L, Corbella X. Effectiveness and safety of an emergency department short-stay unit as an alternative to standard inpatient hospitalisation. Emerg Med J 2006; 23: 833-837.

26. Gómez C, Gulllamont A, Salazar A, Pastor J, Novelli A, Corbella X. Evaluación de la satisfacción de los pacientes ingresados en una unidad de corta estancia de urgencias. Emergencias 2005; 17: 12-16.

27. GonzÁlez-Armengol JJ, Fernández C, MartínSÁNChEz FJ, GonZÁlez-dEl CASTILlo J, LÓPEZ-FARRÉ
A, Elvira C et al. Actividad de una unidad de corta estancia en urgencias de un hospital terciario: cuatro años de experiencia. Emergencias 2009; 21: 87-94.

28. BRIERley S, King D. An emergency department tackles bed management and home-based care. Aust Health Rev 1998; 21: 127-142.

29. Miró O, Salgado E, Bragulat E, Junyent M, Asenjo Sebastián MA, Sánchez M. Estimación de la actividad en urgencies y su relación con la provisión de camas de hopitalización. Med Clin (Barc) 2006; 127: 86-89.

30. Miró O, Salgado E, Bragulat, Ortega M, SalmeRón JM, SÁnchez M. Repercusión de la falta de camas de hospitalización en la actividad de un servicio de urgencias hospitalario. Med Clin (Barc) 2006; 126: 736-739. 